\title{
A Comparison of Venlafaxine and SSRIs in Deliberate Self-poisoning
}

\author{
Agnes N. Chan • Naren Gunja • Christopher J. Ryan
}

Published online: 17 March 2010

(C) American College of Medical Toxicology 2010

\begin{abstract}
To compare the clinical features of deliberate selfpoisoning with venlafaxine and selective serotonin reuptake inhibitors (SSRIs) presenting to the emergency department of an Australian tertiary referral hospital. A retrospective cohort study comparing all 36 patients who presented with venlafaxine self-poisoning with 44 randomly selected patients with SSRI self-poisoning between 1997 and 2006. Patients who had overdosed on venlafaxine were older (mean age 37.4 versus 28.8 years, $p \leq 0.001$ ) and generally exhibited a higher degree of suicidal intent $(p \leq 0.017)$. Median venlafaxine dose taken was 35 defined daily doses (DDDs) compared with SSRIs 19.4 DDDs. Those who ingested venlafaxine
\end{abstract}

Sources of funding: Nil in addition to hospital salary

Previous presentation: Royal Australian and New Zealand College of Psychiatrists Annual Congress, Gold Coast, Australia, May 2007

A. N. Chan $(\bowtie)$

Consultation- Liaison Psychiatry, Royal Prince Alfred Hospital,

Building 92, Level 2, Royal Prince Alfred Hospital,

Camperdown, NSW 2050, Australia

e-mail: agnes.chan@email.cs.nsw.gov.au

A. N. Chan

Psychiatry, University of Sydney,

Sydney, NSW, Australia

N. Gunja $\cdot$ C. J. Ryan

Westmead Hospital,

Westmead, Sydney, NSW 2145, Australia

N. Gunja

NSW Poisons Information Centre, The Children's Hospital,

Westmead, Sydney, NSW 2145, Australia

N. Gunja

Emergency Medicine, University of Sydney,

Sydney, Australia were more likely to become confused $(25 \%$ versus $0 \% ; p=0)$ and have mydriasis $(19.4 \%$ versus $2 \% ; p \leq 0.02)$, than those who took SSRIs. One patient from the venlafaxine group died. Compared with SSRI self-poisoners, patients who deliberately ingested venlafaxine were more likely to exhibit serious suicide intent. They were also more likely to be older, take a higher DDD of the drug, and have confusion and mydriasis. This has implications for management of severely depressed and suicidal patients.

Keywords Venlafaxine - Serotonin reuptake inhibitors · Overdose $\cdot$ Self-poisoning $\cdot$ Suicide

\section{Background}

Venlafaxine is hydroxycycloalkylphenylethylamine-derivative bicyclic antidepressant which inhibits the reuptake of both serotonin and noradrenalin. In the early days of its clinical use venlafaxine was thought to have a toxicity profile similar to that reported for selective serotonin reuptake inhibitors (SSRIs) and was sometimes grouped with the SSRIs in self-poisoning studies $[1,2]$.

More recently, a number of large epidemiological reviews in the UK and Finland have suggested that venlafaxine is associated with more deaths per written prescriptions than other SSRIs suggesting a greater inherent toxicity [3-6]. It is difficult to determine from these large studies whether this is a true toxic effect or one related to the possibility that venlafaxine is prescribed to patients at higher suicidal risk than those prescribed SSRIs [7].

This study aims to compare the demographic details, historical features and clinical sequelae of venlafaxine versus SSRI overdoses presenting to the emergency 
department of a large tertiary referral hospital over a nine year period.

\section{Methods}

This retrospective cohort study was conducted in a large tertiary referral hospital in the western suburbs of Sydney. The hospital's emergency department is serviced by a consultation-liaison psychiatry and toxicology, both of which have maintained a patient database since 1997. All presentations of actual or suspected self-poisoning to the emergency department are referred to consultation-liaison psychiatry and/or toxicology for assessment. The data from the psychiatric assessment done by a psychiatrist, trainee or clinical nurse consultant are entered into the consultationliaison database; a toxicology trainee collects and enters the toxicology data obtained by him/herself or by the toxicologist. The study included all patients who had taken an overdose of venlafaxine between 1997 and 2006 as identified from the databases. A comparison group of 44 SSRI self-poisoning cases was randomly selected from a pool of 257 . The outcome variable for the power calculation was 'expressed intent' (present versus absent). A power analysis determined a sample size of 44 patients who ingested SSRIs would have $80 \%$ power to detect a $25 \%$ difference in intent compared to venlafaxine with a two-tailed alpha of 0.05 . Approval for the study was obtained from the hospital's human research ethics committee.

All variables were extracted by one author (ANC) who was not blinded to the overdose type. The following descriptive variables were extracted from the medical record of each patient: age, sex, relationship status, length of stay, reason for psychiatric referral and referral service, psychiatric diagnoses made, degree of suicide intent, past suicide attempts, psychiatric follow up, amount of antidepressant taken [expressed as defined daily dose (DDD)], concomitantly ingested medications, alcohol or illicit substances, and medical and surgical history. In addition, the following outcome variables were extracted: length of stay in a medical ward; length of stay in the intensive care unit; need for intubation; Glasgow Coma Score on admission; occurrence of seizures or arrhythmia; longest QRS, QT and QTc; highest BP, pulse and temperature; signs of serotonin toxicity according to the Hunter Serotonin Toxicity Criteria [8]; and death.

Degree of suicidal intent was judged from the information presented in the progress notes and recorded according to a five-point scale: no intent or accidental overdose; denied intent, but ambivalent about survival; admitted intent, but ambivalent about dying; admitted intent and made plans to escape detection.

The amount of venlafaxine (DDD) ingested was calculated from amounts recorded in the medical records. The
DDD is based on the assumed average daily dose of the drug when used for its main indication by adults. It is the unit approved by the World Health Organization for drug use studies, and allows for comparisons independent of differences in price, preparation and quantity per prescription [9].

The Hunter Serotonin Toxicity Criteria group features of serotonin abnormalities under three broad areas of mental state (agitation, restlessness, confusion, hypomania), the motor system (clonus/myoclonus, tremor/shivering/hyperreflexia/ hypertonia/rigidity) and the autonomic nervous system (diaphoresis, tachycardia, flushing, mydriasis). Three or more of these features suggest serotonin toxicity [8].

Data were analysed with SPSS for Windows Version 14. Two-tailed tests with a significance level of $5 \%$ were used throughout. No adjustment was made for multiple comparisons. Chi-squared or Fisher's exact tests were used as appropriate to look for differences between patient groups in the distribution of categorical variables. Since many of the continuous variables had highly skewed distribution, Mann-Whitney tests were used for differences between the patient groups.

\section{Results}

Thirty seven cases of venlafaxine deliberate self-poisoning were identified but one of these could not be included as the patient's file was lost. Of the 36 medical records reviewed, one patient coingested venlafaxine and sertraline and was included in the venlafaxine group for the purpose of this analysis. Forty four patients who took an overdose of SSRIs during the study period were randomly identified for inclusion in the study. The SSRIs ingested included sertraline, fluoxetine, citalopram, paroxetine and fluvoxamine.

Descriptive and outcome variables are shown below in Tables 1 and 2, respectively. Continuous, non-normally distributed values are expressed as median values, interquartile ranges and Mann-Whitney $P$ values are used. Continuous, normally distributed values are expressed as mean values, standard deviations and $t$ test $P$ values are used. Categorical values are expressed as nominal values and percentage of the number in the group.

\section{Descriptive Variables}

Table 1 shows the descriptive variables in patients who had ingested venlafaxine or an SSRI. The patients who ingested venlafaxine had a mean age of 37, compared with 29 for the SSRIs patients $(p<0.001)$.

Four cases of venlafaxine ingestion were omitted from the calculation because either the number or strength of the 
Table 1 Descriptive variables in patients who had ingested venlafaxine or an SSRI

\begin{tabular}{|c|c|c|c|}
\hline & $\begin{array}{l}\text { Venlafaxine } \\
n=36\end{array}$ & $\begin{array}{l}\text { SSRI } \\
n=44\end{array}$ & $P$ value \\
\hline Age $($ mean $\pm \mathrm{SD})$ & $37.4 \pm 13.0$ & $28.8 \pm 10.1$ & 0.001 \\
\hline Sex (number of females, as percent of total) & $25(69.4)$ & $31(70.5)$ & 0.992 \\
\hline Amount ingested DDD (median, interquartile values) & $35(10,56)$ & $19.4(7.3,29.5)$ & 0 \\
\hline \multicolumn{4}{|l|}{ Degree of suicide intent (number, as percent of total) } \\
\hline $\begin{array}{l}\text { a. None, accidental } \\
\text { b. Denied, ambivalent }\end{array}$ & $\begin{array}{l}2(5.6) \\
2(5.6)\end{array}$ & $\begin{array}{c}2(4.7) \\
11(25.6)\end{array}$ & \\
\hline c. Intent, but ambivalent & $24(66.7)$ & $28(65.1)$ & \\
\hline d. Intent, plan escape detection & $8(22.2)$ & $2(4.7)$ & \\
\hline $\begin{array}{l}\text { No intent }(\mathrm{a}+\mathrm{b} \text { above }) \\
\text { With intent }(\mathrm{c}+\mathrm{d} \text { above })\end{array}$ & $\begin{array}{r}4(11.1) \\
32(88.9)\end{array}$ & $\begin{array}{l}13(30.2) \\
30(69.8)\end{array}$ & 0.039 \\
\hline No. patients with past suicide attempt (number, as percent of total) & $24(66.7)$ & $22(52.4)$ & 0.201 \\
\hline No. patients with past overdose (number, as percent of total) & $20(47.6)$ & $19(57.6)$ & 0.392 \\
\hline \multicolumn{4}{|l|}{ Psychiatric diagnoses documented (number, as percentage of total) } \\
\hline Adjustment disorder & $9(26)$ & $19(44)$ & 0.099 \\
\hline Major depressive disorder & $19(54)$ & $17(39)$ & 0.183 \\
\hline Dysthymia & $5(14)$ & $7(17)$ & 0.74 \\
\hline Personality disorder & $15(43)$ & $12(27)$ & 0.142 \\
\hline No. patients who co-ingested other antidepressants (number, as percent of total) & $3(8.3)$ & $5(11.4)$ & 0.724 \\
\hline No. patients who co-ingested benzodiazepines (anticonvulsant; number, as percentage of total) & $10(0.28)$ & $11(0.25)$ & 0.779 \\
\hline $\begin{array}{l}\text { No. patients who co-ingested other psychotropics (anticonvulsant and proconvulsan; number, } \\
\text { as percent of total) }\end{array}$ & $5(13.9)$ & $7(15.9)$ & 0.801 \\
\hline No. patients who co-ingested alcohol (number, as percent of total) & $12(33.3)$ & $12(27.3)$ & 0.556 \\
\hline No. patients who co-ingested stimulants (number, as percent of total) & $2(5.56)$ & $2(4.55)$ & 0.889 \\
\hline No. patients who co-ingested depressants (number, as percent of total) & $2(5.56)$ & $2(4.55)$ & 0.889 \\
\hline
\end{tabular}

Venlafaxine $75 \mathrm{mg}=\mathrm{DDD}$

Sertraline $50 \mathrm{mg}=\mathrm{DDD}$

$P$ value of 0.039 is derived from comparing $\mathrm{a}+\mathrm{b}$ vs. $\mathrm{c}+\mathrm{d}$; no expressed intent vs. expressed intent

tablets ingested were unknown and could not be reasonably estimated. The median venlafaxine overdose was 35 DDDs, whereas the median SSRI overdose was 19.4 DDDs.

Among the venlafaxine group 22.2\% (eight of 36) had expressed intent to suicide with plans to escape detection versus $4.7 \%$ (two of 44 ) of SSRI poisoning. In contrast, $25.6 \%$ (11 of 43 ) of SSRI poisoning group denied intent but were ambivalent about the suicide attempt compared with $5.6 \%$ (two of 36 ) of those who took venlafaxine. Overall, the patients who took venlafaxine exhibited a higher degree of intent to die $(p \leq 0.039)$.

\section{Outcome Variables}

Table 2 shows the outcomes of the patients who ingested venlafaxine and SSRIs. The outcome variables to reach a statistically significant difference between the two groups were recorded confusion, mydriasis and longest QT interval.

SSRIs were associated with a significantly longer QT on electrocardiography than venlafaxine. The median longest recorded QT in the SSRI group was $372 \mathrm{~ms}$, while it was only $346 \mathrm{~ms}$ in the venlafaxine group $(p \leq 0.030)$. However, for further analysis, QT was plotted against the heart rate on a QT interval nomogram (Fig. 1) [10]. This showed that neither group demonstrated QT/HR results in the 'at risk' range.

One of the 80 patients included in the study died. This patient had ingested venlafaxine XR 12,600 $\mathrm{mg}$ in an apparently impulsive overdose of ambivalent intent. She had also taken propranolol, alcohol and amphetamine. Her Glasgow coma scale on admission was 14 out of 15 . She subsequently developed seizures, hypotension and ventricular tachycardia. Confusion, mydriasis and diaphoresis were also noted. She died on day 4. A coronial inquest found the cause of death to be related to pericarditis with evidence of superficial myocardial cell necrosis.

\section{Discussion}

Previous studies have suggested that the apparent greater toxicity of venlafaxine may be due to it being prescribed for 
Table 2 Outcome variables in patients who had ingested venlafaxine or an SSRI

\begin{tabular}{|c|c|c|c|}
\hline & $\begin{array}{l}\text { Venlafaxine } \\
n=36\end{array}$ & $\begin{array}{l}\text { SSRI } \\
n=44\end{array}$ & $P$ value \\
\hline Days in a medical ward (median, interquartile values) & $2.0(1.3,2.8)$ & $2.0(1.0,2.0)$ & 0.205 \\
\hline Psychiatric admission (number, as percent of total) & $15(41.7)$ & $13(29.5)$ & 0.258 \\
\hline ICU admission (number, as percent of total) & $4(11.1)$ & $1(2.3)$ & 0.169 \\
\hline Intubation (number, as percent of total) & $3(8.3)$ & $1(2.3)$ & 0.322 \\
\hline Coma recorded (number, as percent of total) & $1(2.8)$ & $1(2.3)$ & 1.000 \\
\hline Seizures recorded (number, as percent of total) & $3(8.3)$ & $1(2.3)$ & 0.322 \\
\hline Arrhythmia recorded (number, as percent of total) & $1(2.8)$ & 0 & 0.45 \\
\hline QRS $>100$ (number, as percent of total) & $8(22.2)$ & $3(6.8)$ & 0.057 \\
\hline Longest recorded QT (mean \pm SD) & $349.2 \pm 37.5$ & $367.7 \pm 34.6$ & 0.030 \\
\hline Longest recorded QTc (mean $\pm \mathrm{SD})$ & $416.5 \pm 24.6$ & $425.3 \pm 24.3$ & 0.126 \\
\hline Highest recorded systolic $\mathrm{BP}$ (mean $\pm \mathrm{SD}$ ) & $123.9 \pm 16.2$ & $128.5 \pm 15.6$ & 0.215 \\
\hline Highest recorded diastolic BP (mean \pm SD) & $72.4 \pm 13.4$ & $70.0 \pm \mathrm{c} 10.8$ & 0.372 \\
\hline Highest recorded pulse (mean \pm SD) & $94.6 \pm 22.1$ & $89.2 \pm 18.7$ & 0.242 \\
\hline Highest recorded temperature (mean $\pm \mathrm{SD}$ ) & $36.5 \pm 0.6$ & $36.7 \pm 0.6$ & 0.283 \\
\hline Confusion recorded (number, as percent of total) ${ }^{\mathrm{a}}$ & $9(25)$ & 0 & 0 \\
\hline Agitation (number, as percent of total) ${ }^{\mathrm{a}}$ & $5(13.9)$ & $3(6.8)$ & 0.457 \\
\hline Restlessness (number, as percent of total) ${ }^{\mathrm{a}}$ & $3(8.3)$ & $1(2.3)$ & 0.322 \\
\hline Hypomania (number, as percent of total) ${ }^{\mathrm{a}}$ & 0 & 0 & - \\
\hline Tremor/shivering (number, as percent of total) ${ }^{\mathrm{a}}$ & $2(5.6)$ & $3(6.8)$ & 1.00 \\
\hline Hyperreflexia/hypertonia/rigidity (number, as percent of total) ${ }^{\mathrm{a}}$ & $8(22.2)$ & $5(11.4)$ & 0.190 \\
\hline Clonus/myoclonus (number, as percent of total) ${ }^{\mathrm{a}}$ & $5(13.9)$ & $4(9.1)$ & 0.724 \\
\hline Diaphoresis (number, as percent of total) ${ }^{\mathrm{a}}$ & $4(11.1)$ & $5(11.4)$ & 1.0 \\
\hline Tachycardia (number, as percent of total) ${ }^{\mathrm{a}}$ & $10(27.8)$ & $7(15.9)$ & 0.197 \\
\hline Flushing (number, as percent of total) ${ }^{\mathrm{a}}$ & 0 & 0 & - \\
\hline Mydriasis (number, as percent of total) ${ }^{\mathrm{a}}$ & $7(19.4)$ & $1(2)$ & 0.02 \\
\hline Serotonin toxicity (number, as percent of total) & $11(30.6)$ & $6(13.6)$ & 0.066 \\
\hline Death (number, as percent of total) & $1(2.8)$ & 0 & 0.45 \\
\hline
\end{tabular}

${ }^{\text {a } H u n t e r ~ S e r o t o n i n ~ T o x i c i t y ~ C r i t e r i a ~}$

patients at higher risk of overdose by virtue of a greater severity of depression [11] and to its inherently greater toxicity $[12,13]$.

There was a higher degree of suicidal intent in venlafaxine self-poisoning than SSRI self-poisoning. The most common diagnosis amongst the venlafaxine self-poisoning group was a major depressive disorder.

In our study, patients who ingested venlafaxine were more likely to be confused and have mydriasis than those who took SSRIs. Howell and co-workers also found a high
Fig. 1 QT Nomogram [10] for venlafaxine poisoning versus SSRI poisoning. The bold line is the 'at risk' line. If the QT/HR point is above the line, the combination is regarded as 'at risk' of developing torsades de pointes

\section{QT Interval Nomogram}

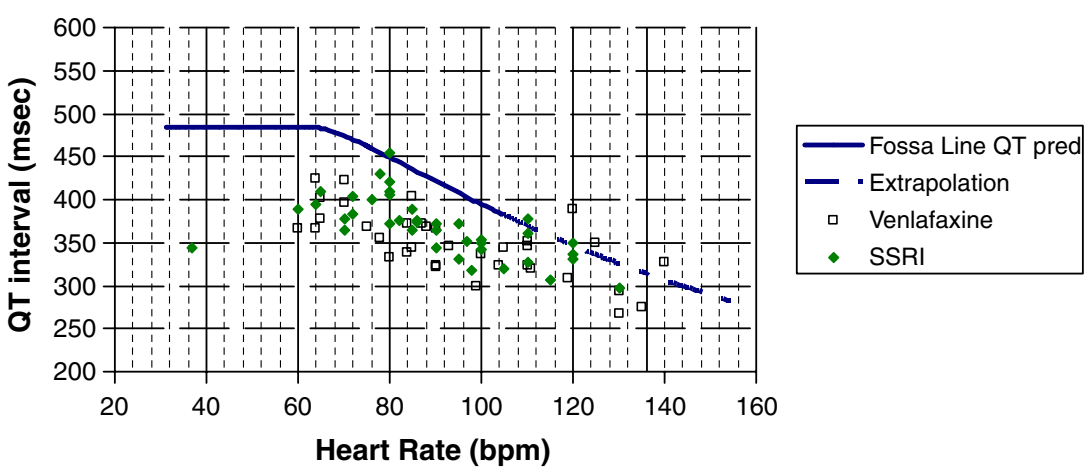


prevalence of mydriasis in patients who took venlafaxine in overdose [14]. Venlafaxine is known to be more toxic in overdose than other SSRIs due to its pro-convulsant activity as well as cardiac sodium channel blocking capacity $[15,16]$.

This is the first comparison study between venlafaxine and the SSRIs to have investigated QT intervals. In spite of the statistical significance in the higher median QT in SSRIs than venlafaxine, we do not feel we can attribute clinical significance to this after further analysis using the QT Interval Nomogram [10]. As illustrated in Fig. 1, most of the QT intervals were below the 'at risk' line for torsades de pointes. There were three QT intervals for venlafaxine and three for SSRIs which were above the 'at risk' line. It is unclear as to whether clinical significance can be attributed to these values as moderate increase in QT prolongation is not necessarily associated with a substantially increased risk of torsades de pointes [17]. The reason for interpreting the QT and not the QTc in our study is that Bazett's formula (QT corrected for heart rate) significantly overcorrects for fast pulse rates and significantly undercorrects for slow pulse rates $[18,19]$. This is a problem in our comparison as venlafaxine overdose is associated with tachycardia; and SSRIs with bradycardia, hence rendering comparison difficult. A retrospective study of venlafaxine poisoning found a dose-dependent relationship with prolonged QTc [14].

The biggest limitation of this study is the small sample size. This may explain why some of the findings from previous studies into the comparative toxicity of venlafaxine and SSRIs, such as the occurrence of seizures, were not replicated. Another significant limitation is co-ingestions. As is often the case in the real world, many of the drug ingestions were polydrug overdoses further confounding any simple comparison of the inherent toxicity of the two types of agent. With the exception of the primary outcome variable, 'expressed intent', all analyses were purely exploratory. Since a large number of comparisons were made, false positive findings are possible and care must be taken in interpreting these results which require confirmation in future independent studies. The study has other limitations. Only one person (ANC) extracted the data from the files and she was not blinded to the type of overdose involved. The study's retrospective nature and the use of routinely collected medical records raise the possibility that salient negative findings might have been overlooked, or assessed but not recorded. Both venlafaxine and SSRI overdose were based on historical evidence alone since serum confirmatory testing is not done routinely.

\section{Conclusion}

This is the first study to look in detail at the psychiatric profile of venlafaxine self-poisoning. We found that patients who deliberately ingested venlafaxine were more likely to exhibit suicide intent. They were also more likely to have confusion and mydriasis.

Our findings lend support to an evolving hypothesis in the literature that venlafaxine may be more toxic than SSRIs in overdose. However, venlafaxine's greater association with completed suicide is partly explained by the fact that it tends to be prescribed to patients at higher risk of a serious suicide attempt.

Acknowledgements Our gratitude to Dr Karen Byth and her assistant Ms Valda Wilson for their statistical input, Dr Rachel Boddy for help with accessing the toxicology database and understanding the Hunter Serotonin Toxicity criteria used, and Dr Geoff Isbister for his comments on ECG parameters and SSRIs toxicity.

\section{References}

1. Rudolph RL, Derivan AT (1996) The safety and tolerability of venlafaxine hydrochloride: analysis of the clinical trials database. J Clin Psychopharmacol 16(suppl 2):54S-61S

2. Baca-Garcia E, Diaz-Sastre C, Saiz-Ruiz J, de Leon J (2002) How safe are psychiatric medications after voluntary overdose? Eur Psychiatr 17:466-470

3. Buckley NA, McManus PR (2002) Fatal toxicity of serotinergic and other antidepressant drugs: analysis of UK mortality data. BMJ, Br Med J 325:1332-1333

4. Morgan O, Griffiths C, Baker A, Majeed A (2004) Fatal toxicity of antidepressants in England and Wales, 1993-2002. Health Stat Q 23:18-24

5. Cheeta S, Schifano F, Oyefeso A, Webb L, Ghodse AH (2004) Antidepressant-related deaths and antidepressant prescriptions in England and Wales, 1998-2000. Br J Psychiatry 184:41-47

6. Koski A, Vuori E, Ojanperä I (2005) Newer antidepressants: evaluation of fatal toxicity index and interaction with alcohol based on Finnish postmortem data. Int J Legal Med 119:344-348

7. Deshauer D (2007) Venlafaxine (Efexor): concerns about increased risk of fatal outcomes in overdose. Can Med Assoc J 176 (1):39-40

8. Dunkley EJ, Isbister GK, Sibbritt D, Dawson AH, Whyte IM (2003) The Hunter Serotonin Toxicity Criteria: simple and accurate diagnostic decision rules for serotonin toxicity. Q J Med 96(9):635-642

9. World Health Organization Collaborating Centre for Drug Statistics Methodology (1999) ATC Index with DDDs. WHO, Oslo

10. Chan A, Isbister GK, Kirkpatrick CMJ, Duffull SB (2007) Druginduced QT prolongation and torsades de pointes: evaluation of a QT nomogram. Q J Med 100(10):609-615

11. Rubino A, Roskell N, Tennis P, Miles D, Weich S, Andrews E (2007) Risk of suicide during treatment with venlafaxine, citalopram, fluoxetine, and dothiepin: retrospective cohort study. BMJ, Br Med J 334:242-247

12. Whyte IM, Dawson AH, Buckley NA (2003) Relative toxicity of venlafaxine and selective serotonin reuptake inhibitors in overdose compared to tricyclic antidepressants. Q J Med 96:369-374

13. Kelly CA, Dhaun N, Laing WJ, Strachan FE, Good AM, Bateman DN (2004) Comparative toxicity of citalopram and the newer antidepressants after overdose. J Toxicol, Clin Toxicol 42(1):67-71

14. Howell C, Wilson AD, Waring WS (2007) Cardiovascular toxicity due to venlafaxine poisoning in adults: a review of 235 consecutive cases. BJCP, Br J Clin Pharmacol 64(2):192-197 
15. Bosse GM, Spiller HA, Collins AM (2008) A fatal case of venlafaxine overdose. Journal of Medical Toxicology 4(1):18-20

16. Khalifa M, Daleau P, Turgeon J (1999) Mechanism of sodium channel block by venlafaxine in guinea pig ventricular myocytes. J Pharmacol Exp Ther 291(1):280-284

17. Isbister GK, Friberg LE, Duffull SB (2006) Application of pharmacokineticpharmacodynamic modelling in management of
QT abnormalities after citalopram overdose. Intensive Care Med 32:1060-1065

18. Balit C, Isbister G, Hackett L (2003) Quetiapine poisoning: a case series. Emerg Med 42(6):751-758

19. Malik M, Camm A (2001) Evaluation of drug-induced QT interval prolongation: implications for drug approval and labelling. Drug Safety 24(323):351 